\title{
Correlation Analysis Between Thyroid Function and Autoantibodies in Hashimoto Thyroiditis Patients with Different lodine Nutritional Status
}

\author{
Li Hongyan ${ }^{1,2}$, Ma Jinqun ${ }^{3}$, Wang Cuicui ${ }^{1}$, Liu Jianfeng ${ }^{3}$, Chen Yunxia ${ }^{3}$, Liu Chunyan ${ }^{3}$, \\ Hou Zhenjiang, ${ }^{2}$, \\ ${ }^{1}$ Department of Medical Technology, Cangzhou Medical College, Cangzhou, China \\ ${ }^{2}$ Institute of Thyroid Diseases Affiliated to Cangzhou Medical College, Cangzhou Thyroid Disease Engineering Technology Research Center, \\ Cangzhou, China \\ ${ }^{3}$ Cangzhou People's Hospital Endocrinology, Cangzhou, China
}

\section{Email address:}

houzhenjiang@sina.com (Hou Zhenjiang)

${ }^{*}$ Corresponding author

\section{To cite this article:}

Li Hongyan, Ma Jinqun, Wang Cuicui, Liu Jianfeng, Chen Yunxia, Liu Chunyan, Hou Zhenjiang. Correlation Analysis Between Thyroid Function and Autoantibodies in Hashimoto Thyroiditis Patients with Different Iodine Nutritional Status. American Journal of Biomedical and Life Sciences. Vol. 9, No. 1, 2021, pp. 10-19. doi: 10.11648/j.ajbls.20210901.12

Received: November 28, 2020; Accepted: January 12, 2021; Published: January 18, 2021

\begin{abstract}
Objective: In order to discuss the correlation between thyroid function stratification and autoantibody titer in HT patients under different iodine nutritional status. Methods: The serum TH, antibody and urinary iodine levels were measured by the electrochemical immune-luminescent apparatus and iodine-catalyzed arsenic-cerium method in 100 HT patients (HT-A, HT-B, HT-C) with different thyroid functions and 60 healthy subjects. Results: The urinary iodine level of HT patients from HT-A increased in turn along with the progression of the disease, while the levels of FT3, FT4, TT3, TT4 decreased gradually and the levels of TSH and thyroid autoantibodies increased gradually. The urinary iodine level of HT-C group was negatively correlated with the serum FT3, FT4, TT3 and TT4 $(P<0.05)$, while the urinary iodine level of HT-B group was negatively correlated with the serum FT3 and TT4 $(P<0.05)$, and positively correlated with TSH $(P<0.05)$. Along with the progression of HT disease, the levels of serum TPOAb and TGAb increased successively, and there were significant differences among each group $(P<0.01)$. The serum TSH level of high TPOAb group and high TgAb group were respectively higher than those of low TPOAb group, low TgAb group and the control group, the levels of FT3 and FT4 were respectively lower than the low TPOAb group and low TGAb group, but there were no statistically significant on the differences between the FT3 of low TgAb and the levels of T3 and T4 of the control group, high TPOAb group, high TGAb group, low TPOAb group and low TGAb group. Conclusions: Under different iodine nutritional status, if the TH and autoantibody levels of HT patients with different thyroid functions changed correspondingly, it could indicate that the iodine nutritional status is involved in the occurrence and development process of HT of different thyroid functions and antibody levels, and plays an important role in it.
\end{abstract}

Keywords: Median Urinary Iodine, Thyroid Hormone, Thyroid Autoantibody, Thyroid Disease, Hashimoto's Thyroiditis

\section{Introduction}

Hashimoto Thyroiditis (HT) is a kind of chronic inflammatory Autoimmune Thyroiditis (AIT) that takes its auto-thyroid tissue as the antigen, it is also named Chronic Lymphocytic Thyroiditis (CLT), which is a common form of AIT and it is also one of the most common types of the
Autoimmune Thyroid Disease (AITD). Patients with HT are mainly characterized by the presence of thyroid-specific autoantibodies, namely anti-thyroid peroxidase antibody (TPOAb), anti-thyroid globulin antibody (TGAb) and thyroid lymphocyte infiltration in the body, which will ultimately lead to the destruction of thyroid tissues and it is the most common cause of hypothyroidism. In recent years, the incidence of HT has been on the rise, and the incidence rate of Hashimoto 
thyroiditis varies from $0.3 \%$ to $10 \%$. The disease can occur in different genders and ages, but mostly in 30 50 years old, especially in middle-aged women, which accounts for about $90 \%$ [1]. There are many pathogenic factors of HT, the environmental, genetic and immune disorder factors can all induce this disease [2], and the pathogenesis of HT is still unclear. In recent years, the correlation between Hashimoto's thyroiditis and other thyroid diseases and thyroid autoantibody levels has been widely studied [3-6]. A large number of epidemiological and clinical studies have been carried out on the relationship between urinary iodine (UI) and thyroid diseases, but the research results are not the same. Taking HT as the research object, there are few studies on the relationship between iodine nutrition level and thyroid function, especially the research on iodine nutrition status in the pathogenesis of HT with different thyroid function and antibody titer. The purpose of this study was to investigate the changes and clinical significance of iodine nutritional status in different thyroid function and antibody levels of HT, and to provide scientific basis for the precise treatment, prevention and pathogenesis of HT.

\section{Datas and Methods}

\subsection{Participants and Grouping}

100 hospitalized HT patients who received outpatient or initial diagnosis without treatment in the Endocrinology Dept. of Cangzhou People's Hospital and Cangzhou Central Hospital during Sept. 2019 to Aug. 2020 were selected as the experimental group, including 26 males and 74 females with an average age of $(47.4 \pm 16.8)$. The patients were divided into normal thyroid function group (HT-A group, 31 cases), subclinical hypothyroidism group (HT-B group, 46 cases) and clinical hypothyroidism group (HT-C group, 23 cases) according to the results of thyroid function examination. 60 cases of healthy physical examination matched with gender and age were selected as the control group (NC group), including 15 males and 45 females with an average age of $(45.8 \pm 17.6)$ years There was no significant difference in gender and age. All the patients were in line with the HT diagnostic criteria in the Chinese Guidelines for the Diagnosis and Treatment of Thyroid Diseases formulated by the Society of Endocrinology, Chinese Medical Association in 2008 [7]. HT Inclusion Criteria: (1) Abnormal serum TSH level, and TPOAb and/or TGAb beyond the upper limit of the normal range; (2) The texture of thyroid is hard, and with or without thyroid enlargement; (3) HT is confirmed by fine-needle aspiration biopsy or Postoperative pathology report. HT Exclusion Criteria: (1) Suffered from systemic lupus erythematosus, rheumatoid arthritis, asthma, pernicious anemia, multiple sclerosis, systemic sclerosis and others recently; (2) Had the history of viral or bacterial infection recently ( $\leq 6$ months); (3) Women with a history of childbirth or pregnancy recently ( $\leq 6$ months); (4) Had the history of malignancy. Standards issued by the World Health Organization (WHO), the United Nations Children's Fund
(UNICEF) and the International Council for the Control of Iodine Deficiency Disorders (ICCIDD) in 2007 were adopted for the assessment of iodine nutritional status. Median urinary iodine (MUI) $<100 \mu \mathrm{g} / \mathrm{L}$ was iodine deficiency, $100 \sim 199 \mu \mathrm{g} / \mathrm{L}$ was iodine adequate, $200 \sim 299 \mu \mathrm{g} / \mathrm{L}$ was iodine excess, $\geq 300$ $\mathrm{g} / \mathrm{L}$ was iodine over-dose [8]. The study was approved by the ethics committee, and all subjects signed informed consent.

\subsection{Research Methods and Projects}

\subsubsection{Specimen Collection}

All subjects were fasted for more than 12 hours. 10 20ml of Mid-stream morning urine samples were collected and stored in a clean polyethylene plastic tube in a refrigerator at $4^{\circ} \mathrm{C}$, and $5 \mathrm{ml}$ of fasting venous blood samples were collected from 8:00 to 10:00. After standing at room temperature for $1 \mathrm{~h}$, centrifugation was performed at $3000 \mathrm{r} / \mathrm{min}$ for $10 \mathrm{~min}$. Some serum samples were taken for determination of thyroid stimulating hormone content and autoantibody level. In the first day of urine retention, avoid eating kelp, laver and other high iodine food and iodine containing drugs, and avoid increasing the amount of drinking water.

\subsubsection{Item Tests}

(1) The levels of serum thyroid stimulating hormone (TSH), Triiodothyronine (TT3), total thyroxineand (TT4), free triiodothyronine (FT3), free thyroxine (FT4), TPOAb and TGAb were measured by the Cobas E601 electrochemical immune-luminescent apparatus of German Roche Company, and the reagents were all the original matching reagents of Roche. Due to the regional differences, as well as the influence of apparatus, reagents and other factors, the reference ranges of each hospital were different. The normal reference ranges of thyroid hormones and autoantibodies in our hospital were as follows: TSH $0.27 \sim 4.2 \mathrm{mIU} / \mathrm{L}$, FT3 2.8 7.1 pmol/L, FT4 12 22 pmol/L, T3 $1.3 \sim 3.10 \mathrm{nmol} / \mathrm{L}$, T4 66 181 nmol/L), TPOAb 0 34IU/ml, TGAb 0 115 IU/ml. (2) The reaction principle of iodine-catalyzed arsenic-cerium was adopted for the measurement of urine iodine [9], the content of urine iodine was measured through the arsenic cerium catalytic spectrophotometry (WS/T107-2006), the kit was produced by Wuhan Zhongsheng Biochemical Technology Co., Ltd., and the apparatus adopted the 7600-110 type automatic biochemical analyzer of Hitachi. (3) Thyroid morphology was examined by the color Doppler ultrasound machine. HT patients mainly presented with a diffuse enlargement of the thyroid gland, thickened blips, uneven distribution and diffuse low-echo.

\subsubsection{Statistical Method}

SPSS 20.0 statistical software was adopted for the statistical analysis, the enumeration data were expressed by rate (\%), its comparison adopted $\chi^{2}$ for testing, the normal distribution measurement data were expressed by mean \pm standard deviation $(\mathrm{x} \pm \mathrm{s})$, and $\mathrm{t}$ was adopted for testing. $P<0.05$ indicated that the difference was considered statistically significant. The measurement data of abnormal distribution were described by median. Kruskal-Wallis $\mathrm{H}$ test was used for 
comparison among multiple groups, Pearson method was used for correlation analysis, and $P<0.05$ was considered as statistically significant.

\section{Results}

\subsection{Comparison of Clinical Features of HT Patients with Different Thyroid Function}

There was no significant difference in gender and age between the two groups $\left(\chi^{2}=2.796, P=0.619\right.$ VS $\chi^{2}=0.912$, $P=0.608)$. Therefore, there is comparability between the research objects (Table 1).

Table 1. Comparison of gender and age of HT patients in each group.

\begin{tabular}{llll}
\hline groups & cases & gender (male, $\mathbf{N}, \%)$ & age (years, $\mathbf{x} \pm \mathbf{s})$ \\
\hline HT & 100 & $26(26.00)$ & $(47.4 \pm 16.8)$ \\
TH-A & 31 & $7(22.58)$ & $(46.1 \pm 17.3)$ \\
TH-B & 46 & $13(28.26)$ & $(47.6 \pm 17.9)$ \\
TH-C & 23 & $6(26.09)$ & $(48.1 \pm 16.7)$ \\
NC & 60 & $15(25.00)$ & $(45.8 \pm 17.6)$ \\
$\chi 2$ & & 2.796 & 0.912 \\
$P$ & & 0.619 & 0.608 \\
\hline
\end{tabular}

\subsection{Comparison of Thyroid Hormones and Autoantibodies in HT Patients with Different Iodine Nutritional Status}

The levels of thyroid hormone and autoantibodies in 100 HT patients with different iodine nutritional status were compared according to the MUI level, Results the MUI of iodine deficiency, iodine adequate, iodine excess and iodine over-dose groups were $89.16 \mu \mathrm{g} / \mathrm{L}, 178.09 \mu \mathrm{g} / \mathrm{L}, 212.69 \mu \mathrm{g} / \mathrm{L}$ and $302.51 \mu \mathrm{g} / \mathrm{L}$ respectively. The constituent ratio of HT patients with different urinary iodine concentrations was the same as that of iodine excess group and iodine over-dose group, which was significantly higher than that of iodine deficiency group. There was negative correlation between MUI and serum FT3, FT4, TT3, TT4 in clinical hypothyroid group $(\mathrm{r}=-0.258, P=0.039 ; \mathrm{r}=-0.296, P=0.029 ; \mathrm{r}=-0.216$, $P=0.043 ; \mathrm{r}=-0.208, P=0.046)$; MUI in subclinical hypothyroid group was negatively correlated with FT3, FT4 (r=-0.289, $P=0.038 ; \mathrm{r}=-0.301, P=0.0349)$, and positively correlated with TSH $(\mathrm{r}=0.295, P=0.028)$. The levels of TSH in iodine deficiency group were higher than those in other three groups. With the increase of urinary iodine level, serum TPOAb and TGAb levels of HT three groups also increased (Table 2).

Table 2. Comparison of thyroid function and serum TPOAb and TG Ab levels in HT patients with different urinary iodine concentrations.

\begin{tabular}{|c|c|c|c|c|c|c|c|c|}
\hline groups & cases & TSH (mIU/L) & FT3 $(\mathrm{pmol} / \mathrm{L})$ & FT4 (pmol/L) & T3 (nmol/L) & T4 (nmol/L) & TPO-Ab (IU/ml) & TG-Ab (IU/ml) \\
\hline iodine deficiency & 9 & $7.82 \pm 1.91$ & $3.94 \pm 1.29$ & $12.16 \pm 2.87$ & $1.68 \pm 0.83$ & $103.8 \pm 21.9$ & $316.8 \pm 291.4$ & $42.81 \pm 36.07$ \\
\hline iodine adequate & 46 & $4.41 \pm 1.26$ & $4.61 \pm 1.26$ & $13.28 \pm 3.12$ & $1.71 \pm 0.62$ & $105.2 \pm 23.6$ & $341.8 \pm 268.3$ & $42.81 \pm 36.07$ \\
\hline iodine excess & 32 & $5.63 \pm 1.41$ & $3.82 \pm 1.31$ & $12.67 \pm 2.96$ & $1.69 \pm 0.71$ & $104 \pm 25.17$ & $461.2 \pm 271.8$ & $76.29 \pm 24.36$ \\
\hline
\end{tabular}

\subsection{Comparison of Thyroid Hormone and Autoantibody Levels in HT Patients with Different Thyroid Functions}

The levels of thyroid hormone and autoantibody in 100 patients with different thyroid function were compared. The results showed that the serum TSH level in HT patients was significantly higher than that in NC patients. With the gradual decrease of thyroid function, TSH levels of HT-A, HT-B and HT-C increased in turn, with the most significant increase of HT-C. Pairwise comparisons between the three groups were statistically significant $(P<0.05)$. The levels of serum FT3 and FT4 were lower in HT and three groups patients of HT than those in NC group, and decreased with the gradual decline of thyroid function of HT-A, HT-B and HT-C. The differences between HT, HT-C and NC were statistically significance $(P<0.05)$, but there was no significant difference between HT-A, HT-B and NC $(P>0.05)$. The serum T3 and T4 levels in HT and every group patients of HT were lower than those in $\mathrm{NC}$ group, but there was no significant difference among the groups $(P>0.05)$. The serum levels of TPOAb and TGAb in HT and every group patients of HT groups were higher than those in NC group $(P<0.001)$. From HT-A, HT-B and HT-C, the levels of TPOAb and TGAb increased successively with the aggravation of the disease, and there were significant difference in different groups $(P<0.01)$ (Table 3$)$.

Table 3. Comparison of thyroid hormone and autoantibody levels in HT patients with different thyroid functions.

\begin{tabular}{|c|c|c|c|c|c|c|c|c|}
\hline groups & cases & TSH (mIU/L) & FT3 (pmol/L) & FT4 (pmol/L) & T3 (nmol/L) & T4 (nmol/L) & TPO-Ab IU/ml & TG-Ab IU/ml \\
\hline HT & 100 & $5.94 \pm 1.61^{\wedge}$ & $4.08 \pm 1.28 *$ & $18.6 \pm 11.37 *$ & $1.79 \pm 0.38$ & $105.49 \pm 19.81$ & $541.2 \pm 236.3^{* \boldsymbol{\Lambda}}$ & $151.32 \pm 68.7 *^{* \boldsymbol{\Lambda}}$ \\
\hline HT-A & 47 & $8.92 \pm 1.67 * *$ & $3.82 \pm 0.98 *$ & $18.02 \pm 8.02 *$ & $1.68 \pm 0.41$ & $106.32 \pm 20.94$ & $241.8 \pm 162.4^{* \boldsymbol{\Delta}}$ & $67.39 \pm 1.27 * \mathbf{\Lambda}$ \\
\hline HT-B & 28 & $11.02 \pm 1.35^{* *}$ & $3.09 \pm 1.04 *$ & $17.36 \pm 7.93^{*}$ & $1.59 \pm 0.52$ & $104.96 \pm 21.62$ & $392.7 \pm 183.2 * \boldsymbol{\Delta}$ & $189.16 \pm 38.7^{* \boldsymbol{\Lambda}}$ \\
\hline $\mathrm{NC}$ & 60 & $2.46 \pm 1.23$ & $4.91 \pm 0.58$ & $19.14 \pm 7.09$ & $1.82 \pm 0.42$ & $104.9 \pm 20.16$ & $9.16 \pm 1.02$ & $15.68 \pm 0.97$ \\
\hline
\end{tabular}

Note: compared with the control group, ${ }^{*}$ was $\mathrm{p}<0.05,{ }^{*} \mathbf{\Lambda}_{\text {was }} \mathrm{p}<0.001$.

\subsection{Comparison of Thyroid Hormone Levels in HT Patients with Different TPOAb and TGAb Levels}

The number of positive cases of TPOAb and TGAb in $100 \mathrm{HT}$ patients were 91 cases and 84 cases, respectively, with the median of $391.27 \mathrm{IU} / \mathrm{ml}$ and $182.59 \mathrm{IU} / \mathrm{ml}$, respectively.
According to this critical value, HT was divided into high TPOAb group, low TPOAb group, high TGAb group and low TGAb group. Comparison of thyroid hormone levels in HT patients with different levels of TPOAb and TGAb, it was found that the serum TSH levels were higher in high TPOAb group and high TGAb group than those of low TPOAb group, low TGAb 
group and control group, FT3 and FT4 levels were lower than those of low TPOAb group and low TGAb group, but the difference of FT3 levels between low TGAb group and control group and the difference of T3 and T4 levels between high TPOAb group, high TGAb group and low TPOAb group, low TGAb group were found no statistical significance (Table 4).

Table 4. Comparison of thyroid hormone levels in HT patients with different TPO-Ab and TG-Ab levels.

\begin{tabular}{lllllll}
\hline groups & n & TSH $(\mathbf{m I U} / \mathbf{L})$ & FT3 $(\mathbf{p m o l} / \mathbf{L})$ & FT4 (pmol/L) & T3 (nmol/L) & T4 (nmol/L) \\
\hline high TPOAb & 54 & $5.63 \pm 1.41^{*}$ & $3.82 \pm 1.31^{*}$ & $12.67 \pm 2.96^{*}$ & $1.69 \pm 0.71$ \\
low TPOAb & 37 & $3.41 \pm 1.26^{*}$ & $4.61 \pm 1.26^{*}$ & $14.28 \pm 3.12^{*}$ & $1.79 \pm 0.62$ \\
high TGAb & 35 & $8.16 \pm 2.59^{*}$ & $3.26 \pm 1.08^{*}$ & $11.83 \pm 2.51^{*}$ & $1.63 \pm 0.65$ \\
low TGAb & 49 & $3.26 \pm 1.48^{*}$ & $4.97 \pm 1.26$ & $13.79 \pm 3.18^{*}$ & $101.3 \pm 19.2$ \\
NC & 60 & $2.46 \pm 1.23$ & $4.91 \pm 0.58$ & $19.14 \pm 7.09$ & $1.71 \pm 0.39$ & $1.82 \pm 0.42$ \\
\hline
\end{tabular}

Note: compared with the control group, ${ }^{*}$ was $\mathrm{p}<0.05$.

\section{Discussion}

Iodine is an essential micronutrient for the synthesis of Thyroid hormone $(\mathrm{TH})$. Studies have shown that iodine accounts for $55 \%$ and $69 \%$ of T3 and T4 respectively [10]. When iodine intake is insufficient or excessive, the synthesis rate and secretion of $\mathrm{T} 3$ and $\mathrm{T} 4$ can be changed, thus affecting the balance of TH. Urinary iodine level is closely related to iodine intake, which is the best index to reflect iodine intake. Fasting urine samples are usually collected to reduce the influence of dietary iodine on the results. Single urine sample can meet the needs of estimating iodine intake. It is unnecessary to use 24-hour urine sample quantification and creatinine correction. Therefore, the early morning fasting urine samples were often collected as the evaluation index of iodine nutritional status [11]. Iodine nutritional status is usually expressed as median urinary iodine (MUI) [8]. A number of epidemiological and clinical datas at home and abroad have shown that urinary iodine levelwas related to thyroid dysfunction. Iodine deficiency or excess can increase the prevalence of clinical and subclinical hypothyroidism and AIT. Shan Zhongyan et al. [12] conducted a cross-sectional epidemiological study of populations with different iodine intakes, which confirmed that both iodine excess and iodine over-dose could significantly increase the prevalence of hypothyroidism and AIT. Teng et al. [13] conducted a five-year prospective follow-up investigation on 3,761 residents in the three regions of Liaoning and Hebei with the MUI of $84 \mu \mathrm{g} / \mathrm{L}, 243 \mu \mathrm{g} / \mathrm{L}$ and $651 \mu \mathrm{g} / \mathrm{L}$ respectively, and found that the incidence of subclinical hypothyroidism in areas with iodine excess and iodine over-dose increased by 11.3 and 12.6 times respectively, and the incidence of AIT respectively increased by 4.4 and 5.5 times. From iodine supplement caused by iodine deficiency to iodine over-dose, this process could accelerate the transformation from subclinical hypothyroidism to clinical hypothyroidism. With the increase of iodine intake, the TSH level of the positive population with thyroid autoantibodies increased significantly. A cross-sectional epidemiological survey of 3,813 residents in two areas on iodine adequate and iodine excess found that the prevalence rate of hypothyroidism and AIT in the iodine excess areas was significantly higher than that in areas with adequate iodine. Li Yushu et al. [14] found that among the $\mathrm{TPOAb}$ positive population in iodine excess and iodine over-dose communities, those whose TSH increased was significantly more than those in the mild iodine deficiency communities, which suggested that the increase of iodine intake may increase the risk of subclinical or clinical hypothyroidism for the people with thyroid autoimmune abnormalities. Guan et al. [15] conducted postpartum follow-up visits for 488 pregnant women and found that the increase of iodine intake could significantly increase the prevalence rate of postpartum thyroiditis, and the prevalence rate of PPT in the iodine sufficient and iodine over-dose groups was respectively 1.6 and 2.6 times than that in the iodine deficient groups. Along with the increase of iodine intake, the prevalence rate of PPT and the positive rate of $\mathrm{TPOAb}$ showed an increasing trend. Pregnant women with high iodine intake had a higher risk of PPT than the women with low iodine intake. Wang Peihua et al. [16] reported that the prevalence rate of hypothyroidism in people with iodine over-dose $(384,000)$ was significantly higher than that in people with iodine adequate $(39,000)$, and the standardized rate was 10.6 times of the iodine adequate. Wu Zhifeng et al. [17] found that the urinary iodine level of 196 patients with thyroid diseases $(306.9 \pm 116.2 \mu \mathrm{g} / \mathrm{L})$ was significantly higher than that of the control group $(195.7 \pm 101.1 \mu \mathrm{g} / \mathrm{L}, P<0.01)$, it shows that iodine over-dose is positively correlated with thyroid diseases. Among them, MUI of subclinical hypothyroidism patients $(363.6 \mu \mathrm{g} / \mathrm{L})$ was higher than that of clinical hypothyroidism $(333.1 \mu \mathrm{g} / \mathrm{L})$. Huang Shuyu et al. [18] analyzed the relationship between urinary iodine level and thyroid disease in 160 newly diagnosed patients with thyroid disease in Xiaogan City, Hubei Province, and found that the order of MUI and the average value from high to low were: hyperthyroidism group (268.0 and $272.1 \pm 18.8 \mu \mathrm{g} / \mathrm{L})$, normal function group (199.7 and $203.7 \pm 16.2 \mu \mathrm{g} / \mathrm{L})$ and hypofunction group $(177.6$ and $185.6 \pm 19.3 \mu \mathrm{g} / \mathrm{L})$. The results showed that the higher the urinary iodine level, the higher the thyroid function, and the urinary iodine level of hypothyroidism was the lowest. Xue Limin et al. [19] reported that the levels of MUI and urinary iodine $(340.73 \mu \mathrm{g} / \mathrm{L}$ and $37.52 \sim 1300 \mu \mathrm{g} / \mathrm{L})$ in patients with thyroid diseases were higher than those in the control group $(198.70 \mu \mathrm{g} / \mathrm{L}$ and $39.76 \sim 778.86 \mu \mathrm{g} / \mathrm{L}$ ), and in patients with different thyroid diseases, the urinary iodine level of subclinical hypothyroidism was the highest $(54.65 \sim 1300.63 \mu \mathrm{g} / \mathrm{L})$, followed by hypothyroidism $(46.52 \sim 1165.85 \mu \mathrm{g} / \mathrm{L})$. Wang 
Fengling et al. [20] found that urinary iodine levels in subclinical hyperthyroidism group, hyperthyroidism group, control group, hypothyroidism group and subclinical hypothyroidism group increased in turn, which suggested that iodine nutrition was related to thyroid function. Subclinical thyroid dysfunction was higher than that in clinical thyroid dysfunction, especially the urinary iodine level of subclinical hypothyroidism was the highest, and the proportion of iodine deficiency and iodine over-dose in clinical and subclinical hypothyroidism patients was higher, and the iodine nutritional composition ratio was quite different. The abnormal rate of thyroid function in the group of urinary iodine $\geq 300 \mu \mathrm{g} / \mathrm{L}$ and $<100 \mu \mathrm{g} / \mathrm{L}$ was higher than that in the group of $100 \sim 199 \mu \mathrm{g} / \mathrm{L}(P<0.05)$, suggesting that iodine over-dose and iodine deficiency can increase the abnormal rate of thyroid function. It suggested that urinary iodine level was more closely related to subclinical hypothyroidism. Although the results of studies on the relationship between MUI and thyroid function were different, the view that iodine intake has an important impact on the spectrum of thyroid diseases was consistent [21]. Wang Yijun et al. [22] reported that the iodine deficiency group of 252 elderly patients with thyroid diseases had statistical significance with the TSH and FT3 in the iodine sufficient or over-sufficient group; the iodine over-dose group had statistical significance with the TSH and FT3 in the iodine sufficient or over-sufficient group; and the three groups had no statistical significance compared with FT4, which suggested that too much or too little iodine intake in the elderly could lead to the increase of thyroid diseases, and the sufficient or over-sufficient iodine intake was very important to maintain the normal thyroid function in the elderly. Na Buqi et al. [23] investigated the relationship of iodine nutritional status and different levels of urinary iodine with thyroid diseases by taking 2,650 physical examination population in Inner Mongolia Autonomous Region as research objects, and the results showed that the levels of urinary iodine were positively correlated with FT4 and FT3, FT4 was negatively correlated with TSH and TgAb, positively correlated with FT3, and positively correlated with TPOAb and TgAb. Ren Yanting et al. [24] found that the serum FT3 and FT4 levels of women in high iodine area were significantly lower than those in appropriate iodine area, while TSH level was significantly increased. The abnormal rate of thyroid function and TSH level in patients with positive autoantibody were significantly higher than those in patients with antibody positive in appropriate iodine area, and the prevalence rate of subclinical hypothyroidism was significantly higher than that in appropriate iodine area. The results showed that the prevalence of subclinical hypothyroidism in fertile women in high iodine areas was higher than that in appropriate iodine areas, and excessive iodine intake and positive thyroid autoantibodies could increase their potential risk. Wang Xinling et al. [25] conducted an investigation on the correlation between thyroid diseases and urinary iodine by adopting the cross-sectional random sampling of 1995 healthy population in Urumqi Municipality, and found that there were no statistically significance on the differences of urinary iodine levels in the thyroid function normal and abnormal groups, as well as in the antibody positive and negative groups $(P>0.05)$, and there was no significant correlation between urinary iodine and TSH and thyroid autoantibodies, which suggested that the levels of urinary iodine did not affect the thyroid function and the expression of autoantibodies, but the relationship between the iodine nutritional status of iodine deficiency, iodine over-dose and most iodine appropriate population and the thyroid function and autoantibodies was not further analyzed. Song Xiangxin et al. [26] stratified the thyroid function of 1,098 inpatients in the Dept. of Endocrinology, People's Hospital of Xinjiang Uygur Autonomous Region, and found that the positive rates of autoantibodies of 221 clinical thyroid dysfunction group were all higher than that of the 316 subclinical thyroid dysfunction groups, but the differences had not statistical significance $(P>0.05)$, and the positive rates of antibodies in each thyroid function abnormal group were significantly higher than that in each thyroid function normal group $(P<0.01)$. In this study, it was found that the urinary iodine and the levels of thyroid autoantibodies of HT patients successively increased in the thyroid function normal group, the subclinical hypothyroidism group and the clinical hypothyroidism group, that is to say, the higher the urinary iodine and the levels of thyroid autoantibodies were, the more serious the degree of damage to thyroid function was in HT patients, this was different from the results of the above studies in which patients with subclinical hypothyroidism had the highest urinary iodine levels, which might be related to the difference between the subjects of study, gender and age and the composition ratio of thyroid diseases, and it needed to be further studied. The results also showed that the serum levels of FT3, FT4, TT3 and TT4 in HT-C group were significantly lower than those in control group and HT-A group, TSH was significantly higher than those in control group and HT-A group, while there was no significant difference in thyroid hormone between HT-A group and control group $(P>0.05)$. TPOAb and TGAb in HT-C group were significantly higher than those in control group and HT-A group, and those in HT-A group were also significantly higher than those in control group, which suggested that the level of thyroid hormone in HT-A patients does not change much, but the levels of TPOAb and TGAb were significantly increased in both HT-C and HT-A patients. It was consistent with the view that high expression levels of autoantibodies could damage thyroid follicles through complement and antibody dependent cell-mediated cytotoxic effect, which eventually led to hypothyroidism. The serum TSH level was significantly increased, FT3, FT4, TT3 and TT4 were decreased, and $\mathrm{TPOAb}$ and TGAb were continuously positive [27].

Epidemiological and clinical studies have been carried out on HT and urinary iodine levels. Wang Yangang et al [28] investigated the relationship between HT and urinary iodine level in Shandong coastal residents by random stratified cluster sampling method. It was found that the $\mathrm{M}$ urine iodine of HT patients $(412.4 \mu \mathrm{g} / \mathrm{L})$ was significantly higher than that of the control group $(291.9 \mu \mathrm{g} / \mathrm{L})$. HT was distributed 
regionally. The closer to the sea, the higher the $M$ urinary iodine of residents, the higher the prevalence of HT. When the M urinary iodine exceeded $300 \mu \mathrm{g} / \mathrm{L}$, the prevalence of HT was significantly increased. It is suggested that high iodine may be the main environmental factors inducing the occurrence and development of HT. Zhang Zhihong et al. [29] reported that the iodine nutrition of the healthy adult population in Jinzhong City, Shanxi Province was in the state of "appropriate amount", while the iodine nutrition of 158 HT patients was obviously in the state of "iodine excess and iodine over-dose", the thyroid functional stratification revealed that the urinary iodine contents were respectively $322.1 \pm 214.2 \mu \mathrm{g} / \mathrm{L}, \quad 332.0 \pm 228.2 \mu \mathrm{g} / \mathrm{L}$ and $252.7 \pm 182.5 \mu \mathrm{g} / \mathrm{L}$ in 35 patients with hyperthyroidism, 42 patients with normal thyroid function and 81 patients with hypothyroidism, there was no significant difference in urinary iodine contents between the thyroid function normal group and the hyperthyroidism group $(P>0.05)$, and there was a significant difference in urinary iodine contents between the thyroid function normal group and the hypothyroidism group and hyperthyroidism group $(P<0.05)$. Li Honghai et al [30] analyzed the urinary iodine levels of 285 patients with different thyroid diseases, and found that the $\mathrm{M}$ urinary iodine levels of HT and hypothyroidism patients $(219.4 \mu \mathrm{g} / \mathrm{L}$ and $214.0 \mu \mathrm{g} / \mathrm{L}$ ) were significantly higher than those of the control group $(164.3 \mu \mathrm{g} / \mathrm{L})$, there was no significant difference between the former two groups. Zhang Dandan et al. [31] found that the M-urine iodine contents of 92 elderly HT patients were respectively $242.73,175.31,97.27 \mu \mathrm{g} / \mathrm{L}$ in the iodine adequate group, the iodine appropriate group and the iodine insufficient group, and the incidence rate of HT in the iodine adequate group was significantly higher than that in the iodine appropriate group and the iodine insufficient group. The urinary iodine of the elderly HT patients $(216.63 \mu \mathrm{g} / \mathrm{L})$ was significantly higher than that of the control group $(179.22 \mu \mathrm{g} / \mathrm{L})$, and the urinary iodine level of the HT hypothyroidism group was higher than that of the HT thyroid function normal group, which confirmed that high iodine could promote the onset of HT. The levels of FT3 and FT4 in the iodine adequate group were significantly higher than those in the iodine appropriate group, while the decreasing of TSH suggested that the excessive iodine intake was related to the onset of the elderly HT, and the high iodine might affect the thyroid function of HT patients. Jin Xin et al. [32] found that the TSH of HT patients was significantly higher than that of the control group, and the FT3 and FT4 of HT patients were significantly lower than that of the control group $(\mathrm{P}<0.05)$. The results of this study showed that in the HT-A group, the MUI was mostly in the iodine appropriate group, and along with the increase of MUI (namely iodine excess and iodine over-dose), the serum FT3, FT4, TT3 and TT4 decreased, and the levels of TSH and serum thyroid autoantibodies gradually increased. The urinary iodine level in HT-C group was negatively correlated with serum FT3, FT4, TT3 and TT4 $(P<0.05)$, while the urinary iodine in HT-B group was negatively correlated with serum FT3 and FT4 $(P<0.05)$, and was positively correlated with TSH $(P<0.05)$. Moreover, in the case of iodine deficiency, the extent of increase of TSH level was more obvious compared with iodine excess and iodine over-dose, which indicated that the change of TSH level was more sensitive to the state of iodine deficiency and was the most sensitive indicator for reflecting the thyroid function. This was basically consistent with the results reported by Wang Fengling and Teng et al. [33, 34] and the opinion that the blood FT3 and FT4 levels of women in areas with high iodine were significantly lower than those in areas with adequate iodine and the TSH levels were higher than those in areas with adequate iodine [24], which suggested that the occurrence and development of HT-B and HT-C were closely related to the deficiency of iodine nutrition and the iodine over-dose. Hypothyroidism could directly reduce the synthesis of FT3 and FT4, and increase the reactive secretion of TSH to compensate for the decreased levels of FT3 and FT4. Therefore, the TSH level was positively correlated with the degree of hypothyroidism and the degree of damage to thyroid tissues [35]. However, there were also reports that there was no significant difference in urinary iodine levels between the thyroid function normal and abnormal groups $(P>0.05)$ [25], and that the FT3 and FT4 of HT patients were higher than those of the control group, while the TSH was lower than that of the control group [31], which might be related to the geographical distribution, different gender, age difference and the different composition ratio of thyroid diseases, and large sample prospective studies were needed to be carried out. Most studies had shown that MUI was closely associated with thyroid diseases. There were significant differences in the urinary iodine levels of HT patients among three groups, which indicated that the high iodine might be a major environmental factor to induce the occurrence and development of HT, and the excessive iodine intake could promote the occurrence of the reaction of thyroid autoimmunity on the one hand, and further promote the occurrence of hypothyroidism in patients with abnormal thyroid autoimmunity on the other hand. It might be that high iodine might aggravate the organic dysfunction of iodine, reduce the synthesis of thyroid hormones, and promote the occurrence of AIT in people with autoimmune background; excessive iodine might lead to the damage of thyroid tissues, accelerate the exposure of thyroid antigens, activate the autoimmune response, and trigger the occurrence of HT [36].

The production of thyroid autoantibodies was closely related to the lymphocyte infiltration of the thyroid, mainly including TPOAb and $\mathrm{TgAb}$, and was an important marker of thyroid autoimmunity. TPOAb was an antibody produced by thyroid micro-some, and the thyroid microsomal antibody (TmAb) detected so far was essentially TPOAb, which had destructive effect after binding with thyroid target tissues. $\mathrm{TGAb}$ was an antibody formed after the thyroglobulin in thyroid follicles entered the blood, which could directly destroy the epithelium of thyroid follicles [37]. The formation of thyroid autoantibodies could further activate the toxic effects of complements and $\mathrm{T}$ cells and induce the sensitized killer $\mathrm{T}$ cells to destroy the function of thyroid follicles. Therefore, the high level TgAb and TPOAb were specific 
markers of HT, which were widely used in the diagnosis of HT [38, 39]. Animal experiments and epidemiological studies show that excessive intake of iodine is closely related to the production of thyroid autoantibodies and the occurrence of AITD. Reducing iodine intake can reduce the incidence rate of AIT $[40,41]$. A large cross-sectional study showed that the positive rates of TPOAb and TGAb and the prevalence of subclinical hypothyroidism in iodine over-dose areas were higher than those in iodine sufficient areas, and the differences were statistically significant [42]. The results of studies on the relationship between thyroid autoantibodies and urinary iodine levels were inconsistent. Sundick et al [43] believed that iodine intake was related to the production of thyroid autoantibodies. Guo Xiaowei et al [44] reported that the median water iodine of school-age children in water-borne high iodine areas were respectively $156.95 \mu \mathrm{g} / \mathrm{L}, 241.26 \mu \mathrm{g} / \mathrm{L}$ and $433.63 \mu \mathrm{g} / \mathrm{L}$, and their MUI were respectively $319.30 \mu \mathrm{g} / \mathrm{L}$, $473.17 \mu \mathrm{g} / \mathrm{L}$ and $764.17 \mu \mathrm{g} / \mathrm{L}$. There was no significant difference in the levels and positive rates of TPOAb and TGAb among the three groups $(P>0.05)$, and there was no statistical significance between the antibody level and positive rate of urinary iodine groups $(P>0.05)$. It was suggested that the level of thyroid autoantibodies in children who drinked high iodine water for a long time was not significantly increased, and subclinical hypothyroidism in children was not related to the level of thyroid antibody positive and TSH. Children may be not the sensitive group of thyroid autoimmunity under high iodine condition. Sha Liping et al [45] conducted a study on the correlation between iodine nutritional status and thyroid diseases in 2827 adults in Ningxia. It was found that the population in this area reached iodine excess. The increase of TPOAb and TGAb had no statistical significance with the normal urinary iodine level and the difference between the groups $(\mathrm{P}>0.05)$, indicating that excess iodine has no effect on the expression of thyroid autoantibodies. Zhang Yaqin et al. [46] studied the relationship between the iodine intake and the thyroid antibodies of 2,593 adults in Hefei, and found that there had no statistical significance on the difference of MUI among TGAb and TPOAb double-positive group (antibody $+/+$ group), either positive group (antibody $+/-$ group) or double-negative group (antibody -/- group), and the multi-factor Logistic regression analysis showed that there was no significant correlation between the urinary iodine level and the production of thyroid autoantibodies, and there was no significant correlation between the iodine over-dose (MUI is $208.59 \mu \mathrm{g} / \mathrm{L}$ ) and the positive of thyroid autoantibodies of the population in Hefei areas. Moulopoulos et al. [47] found that when MUI was at $200 \sim 500 \mu \mathrm{g} / \mathrm{L}$, the stable iodine intake would not cause an increase in the incidence rate of AIT, and this might also be regarded as a reason for the correlation of positive lack between the iodine over-dose and thyroid autoantibodies. Tian Yan et al [48] investigated the iodine nutrition, TSH and thyroid autoantibodies of 2081 adults in Gansu Province, and found that serum TGAb, TMAb levels and positive rate increased with the increase of urinary iodine level, but there was no significant difference in the levels of urinary iodine among groups. It was considered that the increase of urinary iodine level was the cause of thyroid function and morphology changes, but there was no obvious correlation between urinary iodine level and autoantibodies. Ma Ling et al [49] found that TMAb and TGAb were associated with thyroid function injury in HT patients, in which TMAb was positively correlated with TSH $(P<0.05)$ and accompanied by a decrease in FT4, while TGAb was negatively correlated with FT3, FT4 and TT4 $(P<0.05)$, and there was no correlation with TSH $(P>0.05)$. Fang Lu et al [50] analyzed the changes of TPOAb and TGAb levels in 2705 patients with HT. There was no significant difference in the $\mathrm{TPOAb}$ and TgAb levels between women under 50 years old and women over 50 years old $(P>0.05)$. Through the follow-up visits, Li Yushu [51] found that the incidence rates of hypothyroidism were respectively $15.38 \%$ and $8.46 \%$ among those antibody positive people in over-adequate and over-dose iodine intake population during the five years' follow-up visits, which was significantly $1.32 \%$ higher than the iodine deficiency. Once TPOAb and / or TGAb were positive, the antibody could continue to exist even in patients with normal thyroid function. The higher the antibody titer was, the less likely it would disappear spontaneously. The study found that the serum TPOAb and TGAb levels in HT group were higher than those in normal control group, and increased with the increase of MUI. Serum TSH levels in high TPOAb group and high TGAb group were higher than those in low TPOAb group, low TGAb group and the control group, FT3 and FT4 levels were lower than those in low TPOAb group and low TGAb group, but there was no significant difference in FT3 between low TGAb group and control group, T3 and T4 levels in high TPOAb group, high TGAb group and low TPOAb group and low TGAb group. This is basically consistent with the result of Zhu Xudong [52], and verifies Li Yushu's view [51]. The expression of TPOAb and TGAb in HT patients were high, which were closely related to the degree of thyroid function damage, which can reflect the severity of the disease. These results suggested that high levels of TPOAb and TGAb may aggravate the damage of thyroid function in HT patients, suggesting that the risk of thyroid autoantibodies production may be mainly related to autoimmune injury. It has been proved that the increase of iodine intake is a risk factor of hypothyroidism in the patients with autoimmune disorders. In addition, TSH level in antibody positive patients was at the upper limit of normal value, which was also one of the risk factors of hypothyroidism. In addition, the TSH level of antibody positive people at the upper limit of normal value was also one of the hazardous factors for hypothyroidism. In particular, individuals with an autoimmune tendency were sensitive to the iodine excess injury, and the possibility of serum $\mathrm{TgAb}$ positive hypothyroidism was increased. Both iodine over-adequate and iodine over-dose could promote the occurrence and development of thyroid autoimmunity [53].

The results suggest that high iodine intake can affect the secretion of thyroid hormone and the level of autoantibodies in HT patients, and accelerate the development of subclinical 
hypothyroidism to clinical hypothyroidism. It suggested that different iodine nutritional status was closely related to the stratification of thyroid function and the degree of thyroid function damage caused by antibody titer. Monitoring and regulation was of great significance to prevent the transformation of thyroid function in patients with HT and to prevent the changes of HT. It may open up new ideas for the prevention and treatment of HT.

\section{Conclusion}

The level of urinary iodine and thyroid autoantibody in HT patients increased with the progression of the disease, suggesting that the higher the level of urinary iodine and thyroid autoantibody, the more serious the damage of thyroid function in HT patients. The MUI of most HT-A patients was in the iodine appropriate group. Along with the increase of MUI, the serum FT3, FT4, TT3 and TT4 would decrease gradually, while the levels of TSH and serum thyroid autoantibodies would gradually increase. The urinary iodine level of HT-A group was negatively correlated with serum FT3, FT4, TT3 and TT4 $(\mathrm{P}<0.05)$, the urinary iodine level of HT-B group was negatively correlated with serum FT3 and FT4 $(\mathrm{P}<0.05)$, the urinary iodine level of HT-C group was negatively correlated with serum FT3, FT4, TT3 and TT4 $(\mathrm{P}<0.05)$, and the urinary iodine levels of the three groups were positively correlated with TSH $(\mathrm{P}<0.05)$. The TPOAb and TGAb in HT group and each HT group were significantly higher than those in the control group, and along with the progression of HT disease, the levels of serum TPOAb and TGAb would increase successively, and there would have significant differences among each group $(\mathrm{P}<0.01)$. TSH levels in high TPOAb group and high TGAb group were higher than those in low TPOAb group, low TGAb group and control group, FT3 and FT4 levels were lower than those in low TPOAb group and low TGAb grou p. But there was no significant difference in FT3 between low TGAb group and control group, and there was no significant difference in T3 and T4 levels between high TPOAb group, high TGAb group and low TPOAb group, low TGAb group. With the increase of urinary iodine level, TSH, TPOAb and TGAb were highly expressed in HT patients, while serum FT3, FT4, TT3 and TT4 were decreased. It suggested that different iodine nutritional status was closely related to the stratification of thyroid function and the degree of thyroid function damage caused by antibody titer. Regular detection and regulation of urinary iodine may be of great significance for the treatment and prevention of HT.

\section{Acknowledgements}

Cangzhou Key R \& D Plan and Guidance Project (Project No: 1833020011).

Natural Science Foundation of Cangzhou Medical College (No. 18Z015)

\section{References}

[1] Xiang Guangda. Clinical Thyroidology [M]. People's Medical Publishing House, 2013: 200.

[2] Hu S, Rayman MP. Multiple Nutritional Factors and the Risk of Hashimoto's Thyroiditis [J]. Thyroid. 2017, 27 (5): 597-610. doi: $10.1089 /$ thy.2016.0635.

[3] Brčić L, Barić A, Gračan S, et al. Genome-wide association analysis suggests novel loci underlying thyroid antibodies in Hashimoto's thyroiditis [J]. Sci Rep. 2019, 9 (1): 5360. doi: 10.1038/s41598-019.

[4] Zhang Yong xuan. Analysis of the diagnostic value of serum TPO AB, TG AB and TRAb levels in Graves' disease and Hashimoto's thyroiditis [J]. Sichuan J anatomy, 2020, 28 (3): 173-174.

[5] Ma L, Chen YQ, Du J. et al. Correlations between thyroid autoantibodies and thyroid function in patients with newly-diagnosed Hashimoto's thyroiditis [J]. Chin J Mode Med, 2018, 28 (17): 94-97.

[6] Zhang BJ, Zhang J, Li DX. Role of Antithyroid Antibody in Autoimmune Thyroid Disease [J]. China Pharmacist 2020, 23 (11): 2261-2265.

[7] Chinese Guidelines for the Diagnosis and Treatment of Thyroid Diseases-thyroiditis formulated by the Society of Endocrinology, Chinese Medical Association [J]. Chin J inter Med, 2008, 47 (9): 784-788.

[8] Organization W H, UNICEF. Assessment of iodine deficiency disorders and monitoring their elimination: a guide for programme managers. 2nd ed. [J]. Geneva Switzerland Who Department of Nutrition for Health \& Development, 2001.

[9] Zhang Yaping, Yan Yuqin, Liu Liejun, et al. Determination of iodine in urine by ammonium persulfate digestion low arsenic cerium catalytic spectrophotometric method $[\mathrm{J}]$. Chinese Journal of endemic diseases, 2013, 32 (1): 95-100.

[10] Rohner F, Zimmermann M, Jooste P, et al. Biomarkers of nutrition for development--iodine review [J]. J Nutr, 2014, 144 (8): 1322S-1342S.

[11] Chinese Guidelines for the Diagnosis and Treatment of Thyroid Diseases-Iodine deficiency disorders formulated by the Society of Endocrinology, Chinese Medical Association [J]. Chin J inter Med, 2008, 47 (8): 689-690.

[12] Shan ZY, Teng WP, Li YM, et al. Comparative epidemiologic study on the prevalence of iodine-induced hypothyroidism $[\mathrm{J}]$. Chinese Journal of Endocrinology and Metabolism, 2001, 17 (2): 71-74.

[13] Teng WP, Shan ZY, Teng XC, et al. Effect of iodine intake on thyroid diseases in China [J]. N Ensl J Med, 2006, 354 (26): 2783-2793.

[14] Li Yushu, Jin Ying, Teng Weiping, et al. Epidemiological study of thyroid autoantibodies in different areas of iodine intake [J]. Shanghai Journal of immunology, 2002, 22: 91-95.

[15] Guan H, Li C, Li Y, et al. High iodine intake is a risk factor of post-partum thyroiditis: result of a survey from Shenyang, China [J] J Endocrinol Invest [J]. 2005, 28 (10): 876-881. 
[16] Wang Peihua, Zhou Yonglin, Zhang Qinglan, et al. Prevalence of hypothyroidism in residents of iodine excess areas $[\mathrm{J}]$. Chinese Journal of endemic diseases, 2007, 26 (6): 669-672.

[17] Wu Zhifeng, Liang Yuan, Chen Xiaojing. Investigation of urinary iodine in patients with thyroid diseases [J]. Youjiang Medical Journal, 2010, 38 (2): 188-189.

[18] Huang Shuyu, Yan Yimin, Wu Min, et al. Analysis of urinary iodine level of newly diagnosed thyroid disease patients in Xiaogan area $[\mathrm{J}]$. Guangzhou trace element science, 2015, 22 (1): 24-27.

[19] Xue Limin, Liu Geling, Yang Xiaolin, et al. The significance of urinary iodine determination in thyroid diseases [J]. Journal of clinical medical literature, 2017, 4 (43): 8371-8372.

[20] Wang Fengling, Hou Zhenjiang, Liu Yuzhi, et al. Evaluation and analysis of iodine nutritional status in patients with thyroid dysfunction [J]. Chinese medicine guide, 2018, 15 (25): 64-67.

[21] Laurberg P, Cerqueira C, Ovesen L, et al. Iodine intake as a determinant of thyroid disorders in populations [J]. Best Pract Res Clin Endocrinol Metab, 2010, 24 (1): 13-27. doi: 10.1016/j.beem.2009.08.013.

[22] Wang Yijun, Zhou Wei, Yang Chunxiang. The relationship between iodine nutritional status and thyroid disease in the elderly [J]. Journal of Integrated Chinese and Western Medicine on cardiovascular disease, 2018, B6 (5): 98-99.

[23] Na buqi, Zhang Xiaoling, Wang Shuyi, et al. Analysis of thyroid function under different iodine nutritional status [J]. Chinese Journal of endemic diseases, 2019, 38 (3): 235-238.

[24] Ren Yanting, Jia Qingzhen, Zhang Xiangdong, et al. Investigation on thyroid disease of women of childbearing age in different iodine intake areas of Shanxi Province [J]. Chinese Journal of epidemiology, 2014, 35 (1): 45-48.

[25] Wang Xinling, rezwanguli-Usman, Ma Fuhui, et al. Analysis of thyroid function, thyroid autoantibodies and urinary iodine changes of permanent residents in Urumqi, Xinjiang [J]. Chinese Journal of epidemiology, 2015, 36 (8): 811-814 DOI: 10.3760/cma.j.issn.0254-6450.2015.08.

[26] Song Xiangxin, Guo Yanying, Ma Xiaoqing. Analysis of thyroid function and iodine nutritional status of Uygur and Han patients in Xinjiang [J]. Hainan Medical. 2016. 27 (24): 3994-3997.

[27] Brčić L, Barić A, Gračan S, et al. Association of established thyroid peroxidase autoantibody (TPOAb) genetic variants with Hashimoto's thyroiditis [J]. Autoimmunity. 2016; 49 (7) 480-485. doi: 10.1080/08916934.

[28] Wang YG, Yan SL, Zhao SH, et al. Correlation of Hashimoto's thyroiditis with urine iodine level among the coastal districts in Shandong Province [J]. Chinese Journal of Endocrinology and Metabolism, 2004, 20 (4): 337-338.

[29] Zhang Zhihong, Zhang Jinmin, Lian Wenjuan. Study on the relationship between urinary iodine level and Hashimoto's thyroiditis [J]. Basic medicine forum, 2015, 19 (12): 1599-1601.

[30] Li Honghai, Qu Shujun, Li Guangping, et al. Study on urinary iodine level of patients with thyroid disease in Tangshan area $[\mathrm{J}]$. Chinese Journal of health inspection, 2017, 27 (7): 1038-1040.
[31] Zhang Dandan, Shi Nan, Zhang Yan, et al. Correlation between iodine nutrition and SOD, IP-10 and thyroid hormone levels in elderly patients with Hashimoto's thyroiditis [J]. Journal of Chongqing Medical University, 2019, 44 (3): 347-351.

[32] Jin Xin, Shang Xuemei, Chen Haibo, et al. Dynamic changes and significance of regulatory $\mathrm{T}$ cells and Th17 cells in peripheral blood of Hashimoto's thyroiditis [J]. Journal of clinical and experimental medicine, 2018, 17 (19): 2092-2094.

[33] Wang Fengling, Hou Zhenjiang, Liu Yuzhi, et al. Study on the relationship between iodine nutrition level and hypothyroidism [J]. Medical review, 2019, 25 (3): 608-611.

[34] Teng XC, Shi X, Shi XG, Shan ZY, et al. Safe range of iodine intake levels: a comparative study of thyroid diseases in three women population cohorts with slightly different iodine intake levels [J]. Biol Trace Elem Res, 2008, 121 (1): 23-30. doi: 10.1007/s12011-007-8036-0. Epub 2007 Oct 20.

[35] Asik M, Gunes F, Binnetoglu E, et al. Decrease in TSH levels after lactose restriction in Hashimoto's thyroiditis patients with lactose intolerance [J]. Endocrine, 2014, 46: 279-284.

[36] Latrofa F, Fiore E, Rago T, et al. Iodine contributes to thyroid autoimmunity in humans by unmasking a cryptic epitope on thyroglobulin [J]. J Clin Endocrinol Metab, 2013, 98 (11): E1768-1774.

[37] Gong Q, Li X, Gong Q, et al. Hashimoto's thyroiditis could be secondary to vitiligo: the possibility of antigen crossover and oxidative stress between the two diseases [J]. Arch Dermatol Res, 2016, 308 (4): 277-281.

[38] Gabalec1 F, Srbova L, Nova M, et al. Impact of Hashimoto's thyroiditis, TSH levels, and anti-thyroid antibody positivity on differentiated thyroid carcinoma incidence [J]. Endokrynol Pol, 2016, 67 (1): 48-53.

[39] Liang Xiuzhen, Wang Jing, Yang Xiaoqiong, et al. Diagnostic value of thyroid autoantibodies in Hashimoto's thyroiditis: a Meta analysis $[\mathrm{J}]$. International Journal of laboratory medicine, 2018, 39 (10): 1206-1210.

[40] Kolypetri P, Carayanniotis G. Apoptosis of NOD. H2 h4 thyrocytes by low concentrations of iodide is associated with impaired control of oxidative stress [J]. Thyroid. 2014, 24 (7): 1170-1178. doi: $10.1089 /$ thy.

[41] Miranda DM, Massom JN, Catarino RM, et al. Impact of nutritional iodine optimization on rates of thyroid hypoechogenicity and autoimmune thyroiditis: a cross-sectional, comparative study [J]. Thyroid. 2015, 25 (1): $118-124$.

[42] Teng XC, Shan ZY, Chen YY, et al. More than adequate iodine intake may increase subclinical hypothyroidism and autoimmune thyroiditis: a cross-sectional study based on two Chinese communities with different iodine intake levels [J]. Eur J Endocrinol, 2011, 164 (6): 943-950.

[43] Sundick RS, Herdegen DM, Brown TR, et al. The incorporation of dietary iodine into thyroglobulin increases its immunogenicity [J]. Endocrinology, 1987, 120 (5): 2078-2084. doi: 10.1210/endo-120-5-2078.

[44] Guo XW, Liu Y, Zhai LP, et al. Effects of excessive iodine intake on school-age children's health in high water iodine areas $[\mathrm{J}]$. Chinese Journal of Control of Endemic Diseases, 2013, 28 (3): 161-165. 
[45] Sha Liping, Wang Ting, Lu Qingling, et al. Study on the correlation between iodine nutrition level and thyroid disease in Ningxia [J]. Chinese Journal of Endocrinol Metab, may, 2018, 34 (5): 394-397.

[46] Zhang YQ, Chen MW, Wang YM, et al. Characteristics of thyroid autoantibodies and influencing factors among adult population in Hefei Area [J]. Chinese General Practice, 2019, 22 (24): 2991-2995.

[47] Moulopoulos DS, Koutras DA, Mantzos J, et al. The relation of serum T4 and TSH with the urinary iodine excretion [J]. J Endocrinol Invest, 1988, 11 (6): 437-439.

[48] Tian Yan, Liu Jingfang, Tang Xulei, et al. Study on the relationship between iodine nutritional status and TSH, thyroid autoantibodies and thyroid nodules in Gansu Province [J]. Acta nutrica Sinica, 2019, 41 (3): 248-252.

[49] Ma Ling, Chen Yuqiong, Du Juan, et al. Correlation analysis of Hashimoto's thyroiditis autoantibody and thyroid function [J].
Chinese Journal of modern medicine, Journal of Pharmaceutical University, 2018, 28 (17): 94-97.

[50] Fang Lu, Zhu wufei, Liao Xiangyu, et al. Analysis of age, gender distribution and autoimmune antibody level in Hashimoto's thyroiditis [J]. Journal of microcirculation, 2020, 30 (1): 69-72.

[51] Zhu Xudong. Correlation analysis of serum TPOAb, TGAb levels and disease condition in Hashimoto's thyroiditis [J]. 2018, 33 (10): 903-906.

[52] Li Yushu, Zhao Dong, Shan Zhongyan, et al. Epidemiological study of thyroid autoantibodies in different iodine intake areas [J]. Chinese Journal of Endocrinology and metabolism, 2006, 22 (6): 518-522.

[53] Li SM, Xu XL, He YT. Diagnostic and treatment signifi cance of physical examination for postoperative thyroid carcinoma patients and its effect on their hospitalization expenses [J]. Chinese General Practice, 2014, 17 (28): 3353-3355. 